\title{
Electrical transport in C-doped GaAs nanowires: surface effects
}

\author{
Alberto Casadei ${ }^{1}$, Jil Schwender ${ }^{2}$, Eleonora Russo-Averchi ${ }^{1}$, Daniel Rüffer ${ }^{1}$, Martin Heiss ${ }^{1}$, \\ Esther Alarcó-Lladó ${ }^{1}$, Fauzia Jabeen ${ }^{3}$, Mohammad Ramezani $^{1}$, Kornelius Nielsch ${ }^{2}$, \\ and Anna Fontcuberta i Morral ${ }^{*}$

\footnotetext{
${ }^{1}$ Laboratory of Semiconductor Materials, Ecole Polytechnique Fédérale de Lausanne, 1015 Lausanne, Switzerland

${ }^{2}$ Institute of Applied Physics, University of Hamburg, Jungiusstr. 11, 20355 Hamburg, Germany

${ }^{3}$ Laboratory of Quantum Optoelectronics, Ecole Polytechnique Fédérale de Lausanne, 1015 Lausanne, Switzerland
}

Received 16 April 2013, revised 16 May 2013, accepted 17 May 2013

Published online 24 May 2013

Keywords nanowires, doping, surface passivation, III-V semiconductors, GaAs, carrier mobility

${ }^{*}$ Corresponding author: e-mail anna.fontcuberta-morral@epfl.ch

The resistivity and mobility of carbon-doped GaAs nanowires have been studied for different doping concentrations. Surface effects have been evaluated by comparing unpassivated with passivated nanowires. We directly see the influence of the surface: the pinning of the Fermi level and the consequent existence of a depletion region lead to an increase of the mobility up to $30 \mathrm{~cm}^{2} / \mathrm{Vs}$ for doping concentrations lower than $3 \times 10^{18} \mathrm{~cm}^{-3}$. Electron beam induced current measurements show that the minority carrier diffusion path can be as high as $190 \mathrm{~nm}$ for passivated nanowires.

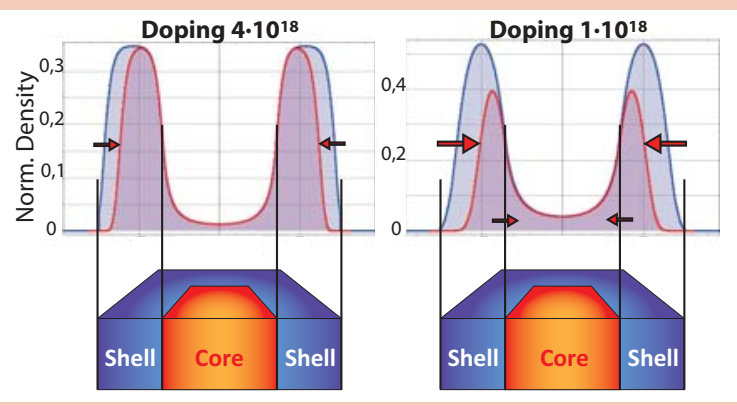

() 2013 WILEY-VCH Verlag GmbH \& Co. KGaA, Weinheim
1 Introduction The particular morphology and dimensions of nanowires (NWs) in the last years have opened many perspectives in applications ranging from electronics, optoelectronics to energy harvesting and storage [1-3]. The successful incorporation of dopants in the nanowires is key for the implementation of such devices. Doping of nanowires has been a challenging task, due to the particular growth mechanisms [4]. In this Letter, we report on the electrical properties of C-doped GaAs nanowires obtained by the Ga-assisted method [5]. As C is not soluble in $\mathrm{Ga}$, its incorporation in the nanowire core through the droplet remains difficult. Our strategy is then to grow an intrinsic NW core and a doped shell around it. The growth of the shell is similar to the thin films growth [6]. We study the doping and surface effects on the NWs resistivity, field effect mobility as well as the minority carrier diffusion length.
2 Method The nanowires consisted of an undoped GaAs core of about $70 \mathrm{~nm}$ in diameter and a C-doped shell. The GaAs nanowires were grown on a (111)Si substrate under conditions that maximize the yield of vertical nanowires, as described elsewhere $[7,8]$ : a rotation of $7 \mathrm{rpm}$, a flux of Ga equivalent to a planar growth rate of $0.3 \AA / \mathrm{s}$, a substrate temperature of $640{ }^{\circ} \mathrm{C}$ and a V/III beam equivalent pressure (BEP) ratio of 50 . Subsequently to the GaAs nanowire synthesis, the growth conditions were radically changed for the obtaining of a $40 \mathrm{~nm}$ GaAs shell. The Ga flux was stopped for about $10 \mathrm{~min}$ while the substrate temperature was lowered to $465^{\circ} \mathrm{C}$ and the V/III BEP ratio was increased to 130 [9]. A p-type doping was achieved by adding a flux of carbon during the growth of the shell. To test the effect of $\mathrm{C}$ doping, we grew a series of samples with different $\mathrm{C}$ flux given by the current applied to the cell, as reported in Table 1. Equivalent samples have been 
Table 1 Doping shell concentration, resistance per unit length and mobility of the nanowires analyzed. "p" corresponds to the passivated NWs and "u" to the unpassivated ones.

\begin{tabular}{llcc}
\hline sample & $\begin{array}{l}\text { doping conc. } \\
\left(10^{18} \mathrm{~cm}^{-3}\right)\end{array}$ & $\begin{array}{l}\text { res./length } \\
(\mathrm{k} \Omega / \mu \mathrm{m})\end{array}$ & $\begin{array}{l}\text { mobility } \\
\left(\mathrm{cm}^{2} / \mathrm{Vs}\right)\end{array}$ \\
\hline $1 \mathrm{p}$ & 8 & 91 & 8 \\
$2 \mathrm{p}$ & 5 & 53 & 12 \\
$3 \mathrm{p}$ & 2.5 & 103 & 14 \\
$4 \mathrm{p}$ & 1 & 2700 & 20 \\
$1 \mathrm{u}$ & 4 & 329 & 8 \\
$2 \mathrm{u}$ & 2 & 300 & 20 \\
$3 \mathrm{u}$ & 1.3 & 528 & 30 \\
\hline
\end{tabular}

fabricated with the addition of a capping layer consisting of $15 \mathrm{~nm} \mathrm{Al}{ }_{0.3} \mathrm{Ga}_{0.7} \mathrm{As}$ and $6 \mathrm{~nm}$ of intrinsic GaAs.

To give an accurate estimate for the resistivity of the nanowires, multiple contacts were fabricated [10]. Multiple four-point measurements have been performed on around 100 samples. The realization of a large number of devices has been automatized thanks to our auto-contacting software [11]. The electrical contacts consisted of $\mathrm{Pd} / \mathrm{Ti} / \mathrm{Au}$ $(40 / 10 / 250 \mathrm{~nm})$ metal layer directly in contact with the doped shell. For the capped nanowires, the $\mathrm{Al}_{0.3} \mathrm{Ga}_{0.7} \mathrm{As}$ shell was removed using a solution of citric acid and hydrogen peroxide $(2: 1)$ leading to an etching rate of $1.4 \mathrm{~nm} / \mathrm{s}$. Bottom gate transistors, with $200 \mathrm{~nm}$ thick $\mathrm{SiO}_{2}$ layer used as a gate dielectric, were realized in order to extract the nanowire majority carrier mobility.

3 Results and discussion Surface states play an extremely important role in the optoelectronic properties of GaAs nanowires. In ambient conditions there is formation of a thin oxide layer on the NW surface resulting in a pinning of the Fermi level. In GaAs the pinning occurs near the middle of the band gap, producing a depletion region. The existence of this region reduces the electrically active part of the NWs [12]. We can avoid this problem by passivating the GaAs with a wider band gap semiconductor, such as AlGaAs $[13,14]$. In this Letter, we study how a passivation layer affects the electronic properties of GaAs NWs with different doping concentrations.

Seven types of samples have been analyzed and the shell doping concentration, the resistance and the mobility are reported in Table 1.

The conductivity $\sigma$ is obtained from four-point measurements and the mobility $\mu$ is derived from bottom gate field effect transistor characteristics:

$$
\mu=-\frac{\partial I}{\partial V_{\mathrm{g}}} \frac{L^{2}}{C \cdot V_{\mathrm{sd}}},
$$

where $V_{\mathrm{g}}$ and $V_{\text {sd }}$ are, respectively, the gate and the sourcedrain voltages, $L$ is the distance between source and drain, and $C$ is the device capacitance. For a structure formed by a nanowire gated through the substrate, it is found [15]:

$$
C=2 \pi \varepsilon_{0} \varepsilon_{\mathrm{r}} \frac{L}{x \cdot \cosh ^{-1}\left(\left(r_{0}+h\right) / r_{0}\right)},
$$

where $\varepsilon_{0} \varepsilon_{\mathrm{r}}$ is the dielectric constant of insulating layer, $r_{0}$ is the NW radius and $h$ the dielectric thickness. In the case of passivated NWs we consider the capping layer as a capacitance in series with the bottom gate dielectric. For $200 \mathrm{~nm} \mathrm{SiO} 2$ thickness with $\varepsilon_{\mathrm{r}}=3.9$ the typical capacitance value of our devices is around $1 \mathrm{fF}$. From the output curves (Fig. 1a) we can clearly see that the carbon acts as $p$ dopant [16].

We assume that all acceptors are ionized at room temperature, meaning that the hole concentration can be directly linked to the $\mathrm{C}$ concentration. As there is no depletion region within the GaAs doped shell of the passivated NWs (1p, 2p, 3p and 4p), we can consider that the electri-
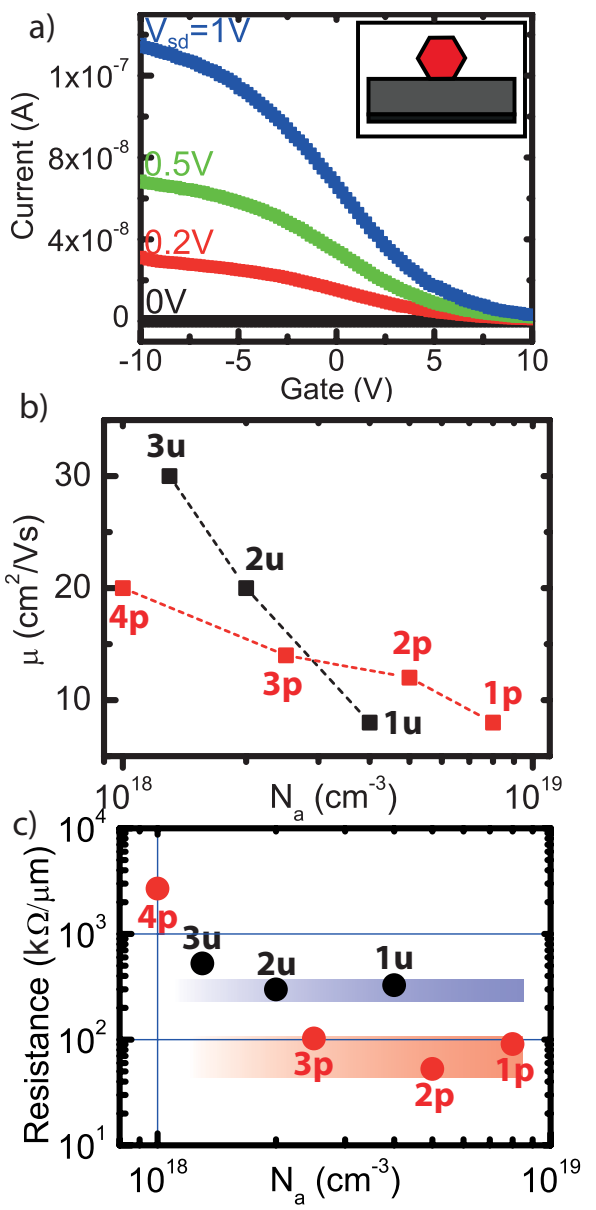

Figure 1 a) Example of a transistor output curve for different source-drain voltages $V_{\text {sd }}$. The inset shows the NW bottom-gate transistor configuration. b) Mobility vs. shell doping concentration for the seven types of NWs analyzed. $1 \mathrm{u}, 2 \mathrm{u}$ and $3 \mathrm{u}$ are unpassivated NWs while $1 p, 2 p, 3 p$ and $4 p$ are passivated. c) NW resistance per length as a function of the doping concentration. The blue and red stripes highlight the region of minimum resistance per length for unpassivated and passivated NWs. 
cally active part corresponds to the entire doped shell. We can therefore introduce the conductivity of the doped shell $\sigma_{\text {shell }}$ calculated on the doped cross-section area and directly extract the doping density as

$$
N_{\mathrm{A}(\text { passivated })}=\frac{\sigma_{\text {shell }}}{\mu \cdot e}
$$

When NWs are not passivated, one needs to consider the Fermi level pinning at the NW surface and the existence of a depletion layer. Solving the Poisson equation and using the boundary conditions of a vanishing electric field at the surface we can calculate the depletion region width. For the doping concentration analyzed, the surface potential $\Phi$ at the GaAs surface is found to be around $0.5 \mathrm{eV}$ [17]. The depletion region $w$ follows [18]:

$$
-\frac{w^{2}}{2}+r_{0} w-\left(r_{0}-w\right)^{2} \ln \left(\frac{r_{0}}{r_{0}-w}\right)=\frac{2 \varepsilon_{\mathrm{r}} \varepsilon_{0} \Phi}{e N_{\text {shell }}}
$$

where $\varepsilon_{\mathrm{r}}=\varepsilon_{\mathrm{GaAs}}=12.9$ is the GaAs dielectric constant and $N_{\text {shell }}$ the doping concentration in the shell. $N_{\text {shell }}$ and $w$ can be deduced from the electrical measurements using the following system:

$$
\left\{\begin{array}{l}
\int_{r_{\text {core }}}^{r_{0}-w} \int_{0}^{2 \pi} N_{\text {shell }} x \sin (\theta) \mathrm{d} x \mathrm{~d} \theta=\frac{L}{\mu R e}, \\
-\frac{w^{2}}{2}+r_{0} w-\left(r_{0}-w\right)^{2} \ln \left(\frac{r_{0}}{r_{0}-w}\right)-\frac{2 \varepsilon_{\mathrm{r}} \varepsilon_{0} \Phi}{e N_{\text {shell }}}=0,
\end{array}\right.
$$

where $R / L$ is the resistance per unit length, $\mu$ is the carrier mobility and $r_{\text {core }}$ is the radius of the undoped nanowire core.

Results on the mobility and resistance per length obtained for all different types of nanowires are shown in Fig. $1 \mathrm{~b}$ and c. The mobility measured increases inversely with the doping concentration for both passivated and unpassivated NWs. This behavior is commonly observed in semiconductors and is related to the scattering of carriers by the doping impurities. Interestingly, we observe a reduction of mobility with the increase in carrier concentration, which results in the saturation of the resistivity for doping concentrations higher than $2 \times 10^{18} \mathrm{~cm}^{-3}$. The saturation values correspond to $\approx 80 \mathrm{k} \Omega / \mu \mathrm{m}$ and $\approx 330 \mathrm{k} \Omega / \mu \mathrm{m}$, respectively, for the passivated and unpassivated NWs.

A priori, one would expect a higher mobility for passivated nanowires with respect to the unpassivated. In Fig. 1b we observe that this is only true for doping concentrations higher than $3 \times 10^{18} \mathrm{~cm}^{-3}$. In order to shed some light on this issue, we have simulated the band profile across the nanowire with nextnano ${ }^{3}$ software (copyright (C2012, nextnano GmbH, Germany) and calculated the carrier distribution inside the NWs. Figure 2 shows the profile of the hole concentration for both passivated and unpassivated NWs and for two doping concentrations:

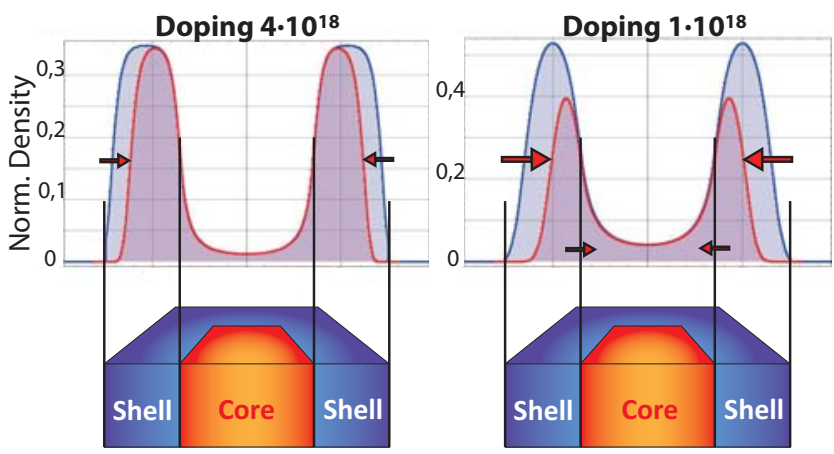

Figure 2 Transverse section view of two NWs with intrinsic core and a doped shell $\left(4 \times 10^{18}\right.$ and $\left.1 \times 10^{18} \mathrm{~cm}^{-3}\right)$. The distribution hole profile is represented in the case of NWs passivated (blue lines) and NWs unpassivated (red lines). In the unpassivated NWs, the depletion region has the effect of keeping the holes far from the surface. The numerical analysis has been performed with nextnano ${ }^{3}$.

$4 \times 10^{18} \mathrm{~cm}^{-3}$ and $1 \times 10^{18} \mathrm{~cm}^{-3}$ (Fig. 2). In the unpassivated case the Fermi level pinning was used.

As shown in Fig. 2 holes are mainly distributed in the doped shell, but a small portion is also found in the core. In the case of unpassivated wires, the surface depletion leads to a decrease in the amount of carriers in the shell. This is especially notable for the low doping concentration. From this we conclude that two mechanisms may contribute to the observation that the highest mobility is for low-doped unpassivated NWs. First, the depletion region in unpassivated and low-doped NWs ensures that transport of carriers occurs at a certain distance from the surface and reduces the surface-related scattering. Second, a higher proportion of the transport occurs through the undoped core, which exhibits highest mobility due to the absence of dopants.

Finally, we used electron beam induced current (EBIC) to study the mean free path of minority carriers. The semiconductor-metal interface at the contacts produces a Schottky barrier. When exciting electron-hole pairs with light or an electron beam, the potential gradient produced by the Schottky barrier attracts minority carriers (electrons) into the contacts, which is measured by an electrical current. The current exponentially decays with the distance from the contact [19]:

$$
I=I_{0} \mathrm{e}^{x / l}+c
$$

where $l$ is the electron mean free path. In order to understand what region of the nanowire is excited with the electron beam, we simulate the trajectory of electrons from the beam with Casino [20], shown in Fig. 3a. We simulate the trajectories of 200 incident electrons with an energy of $3 \mathrm{keV}$ and a probe current of $200 \mathrm{pA}$, corresponding to the experimental conditions used. As it can be seen in Fig. 3a, the excitation is limited to the doped shell of the nanowire.

Figure $3 \mathrm{~b}$ shows the exponential decay of the current for an unpassivated and two passivated NWs with hole 


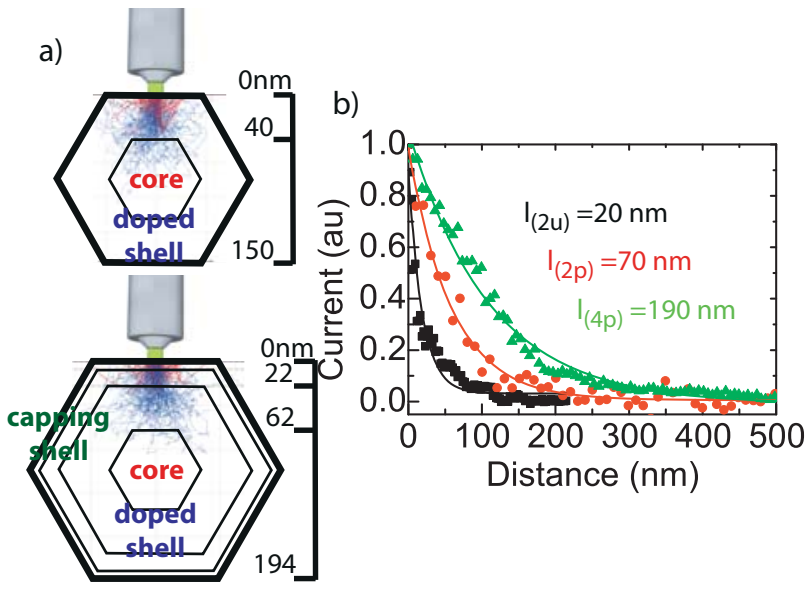

Figure 3 a) Electron trajectory in the NWs simulated by Monte Carlo. The simulations are performed for 200 electrons with energy of $3 \mathrm{keV}$ and a probe current of $300 \mathrm{pA}$. b) Experimental current decay as a function of distance to the contact for three NW samples ( $2 u, 2 p$ and $4 p)$. The minority carrier mean free path extracted increases up to $190 \mathrm{~nm}$ for passivated NWs with a doping concentration of $10^{18} \mathrm{~cm}^{-3}$.

concentrations, respectively, of 2, 4 and $1 \times 10^{18} \mathrm{~cm}^{-3}$. The lowest mean free path is $20 \mathrm{~nm}$ for the unpassivated nanowire. The passivated nanowires exhibit longer mean free paths for minority carriers, up to $190 \mathrm{~nm}$. The results are in perfect agreement with the work done by Chang et al. on analogue NWs [21]. Despite the majority carrier (holes) mobility is lower for passivated NWs, a longer diffusion path is found for the minority carriers (electrons). This is explained again by the Fermi level pinning at the surface and its respective electric field, which attracts the beaminduced electrons towards the surface. Consequently, the electron mean free path in unpassivated NWs is reduced by the larger defect and trap densities at the surface.

4 Conclusions In conclusion, we have measured the carrier concentration, mobility and minority diffusion length for core-shell GaAs nanowires where the shell is $\mathrm{C}$-doped at different concentrations. The effect of the surface states is evaluated by comparing the values for passivated and unpassivated NWs. At low doping concentrations the highest mobility is observed for unpassivated nanowires, while at higher doping concentrations the passivated nanowires offer the best characteristics. The highest mobility $\left(30 \mathrm{~cm}^{2} / \mathrm{Vs}\right)$ was obtained for unpassivated NWs with a doping concentration of $1.3 \times 10^{18} \mathrm{~cm}^{2} / \mathrm{Vs}$. Finally, we have measured the minority carrier diffusion lengths up to $190 \mathrm{~nm}$ for passivated NWs with a doping concentration of $10^{18} \mathrm{~cm}^{2} / \mathrm{Vs}$.

Acknowlegements The authors thank financial support from: The Swiss National Science Foundation under Grant
Nos. 2000021-121758/1 and 129775/1; NCCR QSIT; The European Research Council under Grant "Upcon".

\section{References}

[1] K. Tomioka, M. Yoshimura, and T. Fukui, Nature 488(7410), 189-192 (2012).

[2] J. Wallentin, N. Anttu, D. Asoli, M. Huffman, I. Aberg, M. Magnusson, G. Siefer, P. Fuss-Kailuweit, F. Dimroth, B. Witzigmann, H. Xu, L. Samuelson, K. Deppert, and M. Borgstrom, Science 339(6123), 1057-1060 (2013).

[3] P. Krogstrup, H. I. Jørgensen, M. Heiss, O. Demichel, J. V. Holm, M. Aagesen, J. Nygard, and A. Fontcuberta i Morral, Nature Photon. 7(4), 306-310 (2013).

[4] J. Wallentin and M. Borgström, J. Mater. Res. 26, 21422156 (2011).

[5] C. Colombo, D. Spirkoska, M. Frimmer, G. Abstreiter, and A. Fontcuberta i Morral, Phys. Rev. B 77, 155326 (2008).

[6] H. Ito and T. Ishibashi, Jpn. J. Appl. Phys. 30, L944-L947 (1991).

[7] E. Russo-Averchi, M. Heiss, L. Michelet, P. Krogstrup, J. Nygard, C. Magen, J. Ramon Morante, E. Uccelli, J. Arbiol, and A. Fontcuberta i Morral, Nanoscale 4, 1486-1490 (2012).

[8] E. Uccelli, J. Arbiol, C. Magen, and P. Krogstrup, Nano Lett. 11, 3827-3832 (2011).

[9] M. Heigoldt, J. Arbiol, D. Spirkoska, and J. Rebled, J. Mater. Chem. 19, 840 (2009).

[10] J. Dufouleur, C. Colombo, T. Garma, B. Ketterer, E. Uccelli, M. Nicotra, and A. Fontcuberta i Morral, Nano Lett. 10(5), 1734-1740 (2010).

[11] P. Blanc, M. Heiss, C. Colombo, A. Dalmau Mallorquì, T. Saberi Safaei, P. Krogstrup, J. Nygard, and A. Fontcuberta i Morral, Int. J. Nanotechnol. 10(5-7), 419-432 (2013).

[12] M. Heiss, C. Colombo, and A. F. Morral, Proc. SPIE 8106 (Nanoepitaxy: Materials and Devices III), 810603 (2011); DOI: $10.1117 / 12.896471$

[13] H. Demers, N. Poirier-Demers, D. Drouin, and N. de Jonge, Microsc. Microanal. 16(6), 795 (2010).

[14] K. Tateno, H. Gotoh, and Y. Watanabe, Appl. Phys. Lett. 85(10), 1808 (2004).

[15] D. R. Khanal and J. Wu, Nano Lett. 7, 2778-2783 (2007).

[16] N. Han, F. Wang, J. J. Hou, F. Xiu, S. Yip, A. T. Hui, T. Hung, and J. C. Ho, ACS Nano 6(5), 4428-4433 (2012).

[17] G. Landgren, R. Ludeke, Y. Jugnet, J. F. Morar, and F. J. Himpsel, J. Vac. Sci. Technol. B 2(3), 351 -358 (1984).

[18] A. Casadei, P. Krogstrup, M. Heiss, and J. Rohr, Appl. Phys. Lett. 102, 013117 (2013).

[19] J. E. Allen, D. E. Perea, E. R. Hemesath, and L. J. Lauhon, Adv. Mater. 21(30), 3067-3072 (2009).

[20] D. Drouin, A. R. Couture, D. Joly, X. Tastet, V. Aimez, and R. Gauvin, Scanning 29(3), 92-101 (2007).

[21] C. C. Chang, C. Y. Chi, M. Yao, N. Huang, C. C. Chen, J. Theiss, A. W. Bushmaker, S. LaLumondiere, T. W. Yeh, M. L. Povinelli, C. Zhou, P. D. Dapkus, and S. B. Cronin, Nano Lett. 12(9), 4484-4489 (2012). 